\title{
Del brochure al paciente: rol de la simulación en el uso de nuevos implantes ortopédicos
}

\author{
From the "brochure" to the patient: role of simulation \\ in the use of new orthopedic implants
}

\author{
Julio J Contreras, * Rodrigo Liendo, * Rodrigo de-Marinis, * \\ Claudio Calvo, ${ }^{*}$ Francisco Soza*
}

Palabras clave: Simulación, ortopedia, traumatología, implante.

Keywords: Simulation, orthopaedics, traumatology, implant.

\footnotetext{
* Departamento de Traumatología y Ortopedia. Pontificia Universidad Católica de Chile, Santiago, Chile
}

Recibido: $15 / 04 / 2021$ Aceptado: 20/05/2021

doi: 10.35366/101431

\section{RESUMEN}

Introducción: El desarrollo de la cirugía ortopédica ha llevado a una constante producción de nuevos implantes. La simulación es una herramienta clave en el uso de nuevos implantes en cirugía ortopédica. El objetivo de este manuscrito es describir detalladamente el proceso ideal de la transferencia desde el instructivo del nuevo implante brochure hasta la aplicación directa en un paciente a través de un boot camp. Material y métodos: Se plantea una modalidad de trabajo para la recepción de nuevos implantes de ortopedia y traumatología basada en la modalidad boot camp. El proceso involucra el análisis teórico del implante, realizando una simulación en huesos y articulaciones artificiales, y finalmente, aplicando esto a la práctica en laboratorio cadavérico. Resultados: Se describe una experiencia piloto de esta modalidad de trabajo en relación con un caso clínico real para la aplicación de un implante nuevo para fracturas de húmero proximal denominado AFFIXUS ${ }^{\circledR} \mathrm{Na}$ tural Nail ${ }^{\circledR}$ de la empresa BIOMET ${ }^{\circledR}$. Conclusiones: Este boot camp es una forma segura para obtener habilidades técnicas específicas de la cirugía ortopédica, sin poner en riesgo a los pacientes. El hecho de establecer un sistema estructurado para la evaluación de nuevos implantes en ortopedia y traumatología es novedoso.

\section{ABSTRACT}

Introduction: The development of orthopedic surgery has led to a constant production of new implants. Simulation is a key tool in the use of new implants in orthopedic surgery. Objective: To describe in detail the ideal transfer process from the new implant instructions "brochure" to direct application to a patient through a "boot camp". Material and methods: A work modality are proposed for the reception of new orthopedic and trauma implants based on the "boot camp" modality. The objective is to carry out a process of theoretical analysis of the implant, leading to simulation in artificial bones and joints, and finally, to the cadaveric laboratory practice. Results: A pilot experience of this work modality is described in relation to a real clinical case for the application of a new implant for proximal humerus fractures called AFFIXUS ${ }^{\circledR}$ Natural Nail ${ }^{\circledR}$ from the company BIOMET ${ }^{\circledR}$. Conclusions: This "boot camp" is a safe way to obtain specific technical skills of orthopedic surgery, without putting surgeons or patients at risk. The fact of establishing a structured system for the evaluation of new implants in orthopedics and traumatology is novel.

\section{INTRODUCCIÓN}

E desarrollo acelerado de la cirugía ortopédica ha llevado a una constante producción de nuevos implantes. ${ }^{1}$ Aunque la osteosíntesis fue realizada por primera vez en el siglo XIX, ${ }^{2}$ el uso generalizado de implantes ortopédicos se aceleró después de la primera artroplastia exitosa a fines de la década de 1950. ${ }^{3}$ En los últimos años, la producción de nuevos implantes ha crecido exponencialmente. ${ }^{1,4}$
El uso de nuevos implantes obliga muchas veces a los cirujanos ortopédicos a realizar una transferencia rápida y directa al paciente. Los cirujanos con experiencia logran minimizar la curva de aprendizaje. ${ }^{5}$ Sin embargo, la transferencia directa no está libre de complicaciones. El uso de nuevos instrumentales enlentece el proceso quirúrgico, lo que lleva a mayor riesgo de infección y complicaciones. ${ }^{6,7}$

Las condiciones laborales son exigentes desde el punto de vista profesional y médico-legal; el

Citar como: Contreras JJ, Liendo R, de-Marinis R, Calvo C, Soza F. Del brochure al paciente: rol de la simulación en el uso de nuevos implantes ortopédicos. Rev Latinoam Simul Clin. 2021; 3 (2): 74-79. https://dx.doi.org/10.35366/101431 
enfoque en la seguridad del paciente y judicialización médica convierte la transferencia directa en un acto riesgoso. ${ }^{8}$ Los cirujanos son medidos y las expectativas de los pacientes han aumentado. ${ }^{9}$

La simulación es una herramienta clave en el uso de nuevos implantes en cirugía ortopédica. ${ }^{10}$ La comunidad ortopédica se ha entrenado utilizando huesos y articulaciones artificiales (Sawbone $\left.{ }^{\circledR}\right)$. Adicionalmente, lo nuevo en simulación incluye campamentos de entrenamiento llamados boot camps, entrenamientos en cirugía cadavérica y simuladores de realidad virtual. ${ }^{11}$

En este contexto, el objetivo de este manuscrito es describir detalladamente el proceso ideal de la transferencia desde el instructivo del nuevo implante brochure hasta la aplicación directa en un paciente, pasando por un entrenamiento simulado de tipo boot camp.

\section{MATERIAL Y MÉTODOS}

Se plantea una modalidad de trabajo para la recepción de nuevos implantes de Ortopedia y Traumatología basada en la modalidad boot camp. Se realizará un proceso de análisis teórico del implante, aplicado posteriormente a la simulación en huesos y articulaciones artificiales, y finalmente, transferir esto a la práctica en laboratorio cadavérico. Idealmente, se plantea el campamento de entrenamiento con duración de una semana previa a la necesidad de utilizar el nuevo implante en una cirugía real, con 24 horas de duración para cada paso estipulado. Esto permite coordinar adecuadamente la obtención de material artificial y cadavérico. El boot camp para nuevos implantes presenta ocho pasos generales que serán detallados a continuación:

1. Revisión de brochure (instructivo) del nuevo implante: se enfoca en detalles clínicos, del implante y del acto quirúrgico. Primero, se evalúan las indicaciones recomendadas por el diseñador para el uso del implante con base en la evidencia disponible y la experiencia de los cirujanos. Segundo, se evalúan las características del implante en relación con material, dimensiones, instrumental y número de piezas. Tercero, se evalúa la técnica quirúrgica recomendada por el diseñador y se contrasta con la técnica habitual utilizada por los cirujanos evaluadores. Finalmente, se realiza una lista de cambios del diseño mayores y menores, para evaluar su sustento en la literatura médica disponible, y definir ventajas/desventajas reales del implante en contraste con modificaciones sin efecto realizadas con tal de dar identidad única al nuevo implante.

2. Revisión bibliográfica de los cambios de diseño mayores y/o menores: en el segundo día del campamento de entrenamiento, se realiza la búsqueda bibliográfica dirigida con relación a los cambios de diseños mayores y menores reportados por el fabricante y encontrados por los cirujanos en el primer día. Los hallazgos son presentados en reunión para discusión y definición por parte de los cirujanos de las verdaderas ventajas/desventajas del implante y las posibles indicaciones ideales o contraindicaciones basadas en la búsqueda bibliográfica y experiencia de los cirujanos. Los acuerdos de esta discusión son plasmados en el acta del campamento.

3. Discusión de aspectos positivos y/o negativos del nuevo implante: los acuerdos son revisados y se realiza un análisis definitivo de los aspectos positivos y negativos del implante. Una vez discutidos en forma detallada, el campamento de entrenamiento puede terminar el tercer día con un veredicto negativo del implante, decidiendo cambiar el manejo definitivo del paciente, suspendiendo la actividad de simulación hasta presentar un caso ideal según los criterios expuestos previamente y dejando registro de todos los acuerdos respecto al uso del nuevo implante.

4. Planificación "paso a paso" de la técnica quirúrgica: en caso de obtener un veredicto positivo en la aplicación del nuevo implante en la cirugía programada, se realiza detalladamente el "paso a paso" de la cirugía, analizando posibles dificultades y técnicas para disminuir al máximo las complicaciones intraoperatorias.

Este "paso a paso" se detalla en forma escrita y gráfica a modo de presentación, con tal de dejar un registro útil para nuevas cirugías. En este paso se revisan videos de técnica quirúrgica que aporta la industria de implantes.

5. Revisión de instrumental y ensamblaje de componentes: se realiza una revisión exhaustiva del instrumental y forma de ensamblaje de éstos y de los implantes. Se registran pasos críticos y se complementa el "paso a paso" realizado el cuarto día de la actividad. En este paso, es clave la participación de la empresa para resolver dudas del brochure respecto a instrumental, disponibilidad en la 
región de algunos elementos del implante $y / 0$ instrumental y muchas veces se incluye a los instrumentistas quirúrgicos que participarán del acto quirúrgico.

6. Simulación con nuevo implante en huesos y/o articulaciones artificiales: se selecciona el hueso y/o articulación artificial ideal (anatomía, material) para la simulación del acto quirúrgico. Además, se planifica una fijación de éste lo más similar al acto quirúrgico, con tal de mejorar la experiencia háptica y propioceptiva y lograr una simulación efectiva. Se vuelve a revisar el instrumental, pero esta vez en ejecución y se identifican nuevos pasos críticos, los cuales son agregados a los dos pasos previos.

7. Simulación con nuevo implante en material cadavérico y acto quirúrgico definitivo: la simulación cadavérica es el acto más cercano a la cirugía real. Permite la simulación de los diversos abordajes y mejorar la experiencia en relación con el manejo de las partes blandas asociadas al procedimiento. Se realiza disección anatómica necesaria para identificar potenciales riesgos de lesión de estructuras circundantes (nervios, vasos sanguíneos, tendones). Se realiza una evaluación detallada para encontrar aspectos no identificados en cualquiera de los pasos previos. Se realiza el acto quirúrgico definitivo buscando emular todas las condiciones planteadas en la simulación con tal de generar comodidad y confianza al equipo operador. Se solicita siempre de respaldo el implante habitual para resolver la patología por tratar con tal de tener un plan
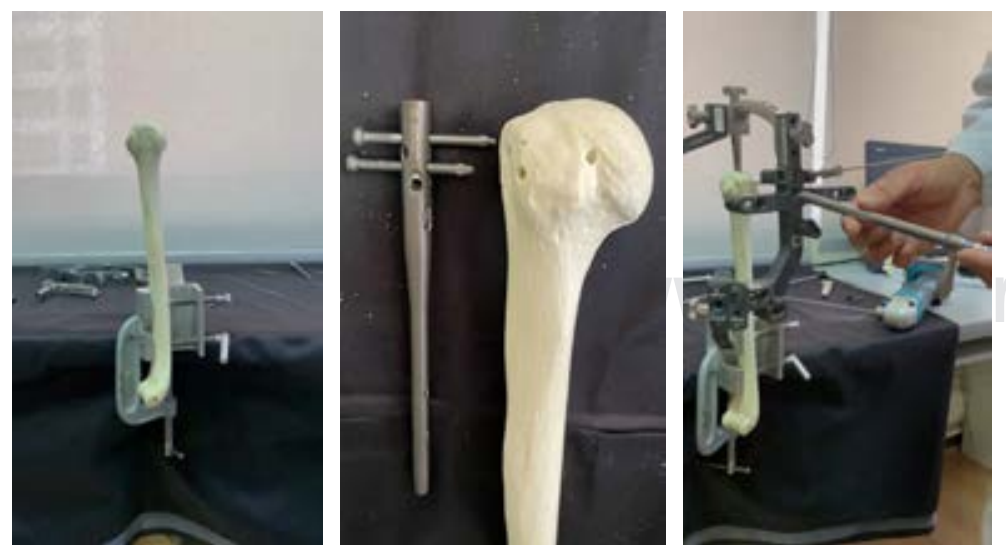

Figura 1: Simulación con nuevo implante en huesos y/o articulaciones artificiales. de respaldo en caso de encontrar desafíos intraoperatorios no predecibles en los pasos previos.

8. Evaluación crítica de los primeros casos de cirugía real y evaluar necesidad de volver a pasos previos: se realiza una evaluación crítica por el equipo quirúrgico y por pares con tal de definir las ventajas/desventajas llevadas al acto quirúrgico verdadero y se define la utilización posterior del implante en éste y/u otro tipo de casos. Se registran todas las observaciones para complementar todos los pasos previos. Se realizará una experiencia piloto de esta modalidad de trabajo en relación con un caso clínico real para la aplicación de un implante nuevo para fracturas de húmero proximal denominado AFFIXUS ${ }^{\circledR}$ Natural Nail ${ }^{\circledR}$ de la empresa BIOMET $^{\circledR}$. Para esto, se utilizó la pauta de trabajo planteada y se realizaron dos casos quirúrgicos reales.

\section{RESULTADOS}

1. Revisión de brochure (instructivo) del nuevo implante: se evaluó la técnica quirúrgica recomendada y se contrastó con la técnica habitual. Se definió que en casos abiertos se optaría por un mini deltopectoral proximal y en casos percutáneos por una entrada anterior a la articulación acromioclavicular.

2. Revisión bibliográfica de los cambios de diseño mayores y/o menores: la evidencia médica disponible se enfocó en la presencia del tornillo de calcar con posibilidad de bloqueo. ${ }^{12}$ Los hallazgos fueron presentados en reunión para discusión y definición por parte de los cirujanos y fueron registrado en la pauta.

3-5. Se realizó la discusión de aspectos positivos y/o negativos del nuevo implante, la planificación "paso a paso" de la técnica quirúrgica y la revisión de instrumental y ensamblaje de componentes de la forma planificada.

6. Simulación con nuevo implante en huesos y/o articulaciones artificiales: se usó un húmero completo de la empresa Sawbone ${ }^{\circledR}$ para la simulación del acto quirúrgico (Figura 1). Se realiza el acto quirúrgico "paso a paso" y se vuelve a revisar el instrumental, identificando nuevas dificultades en la simulación al no presentar partes blandas.

7. Simulación con nuevo implante en material cadavérico y acto quirúrgico definitivo: se 

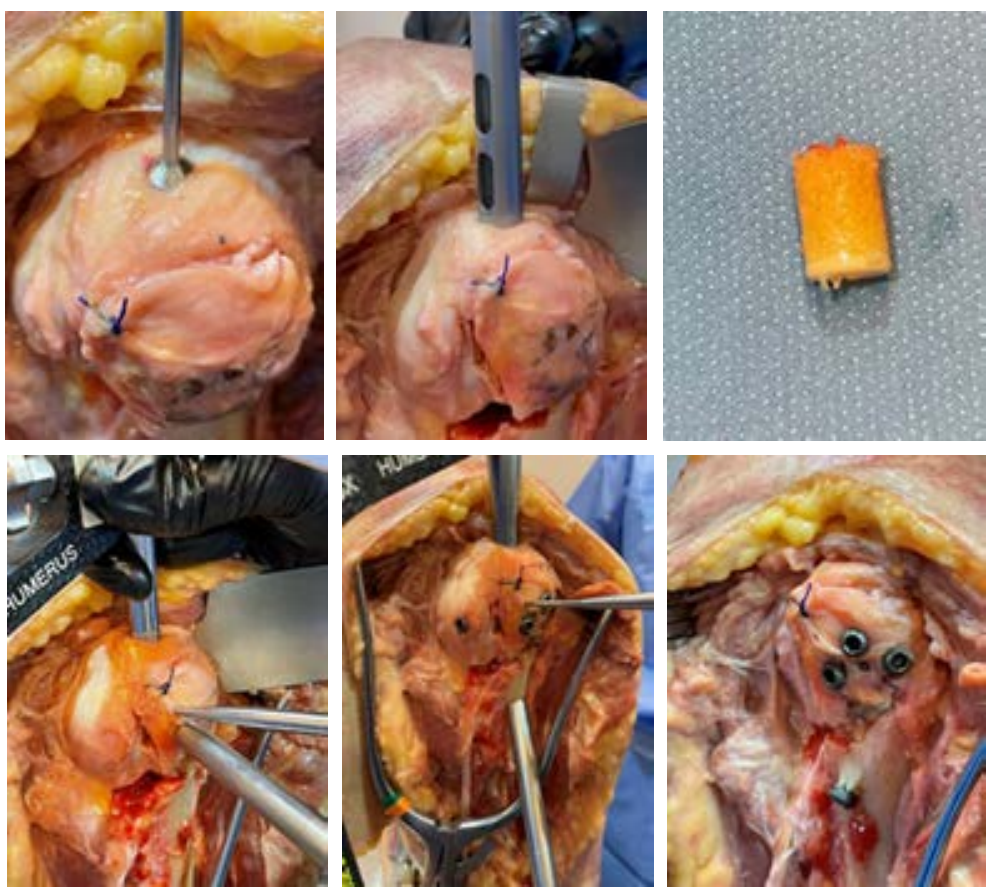

Figura 2: Simulación con nuevo implante con material cadavérico.

realizó la simulación del acto quirúrgico en una extremidad superior desarticulada a nivel de escápula (Figura 2). Se colocó en la posición habitual de cirugía y se realizó un abordaje deltopectoral proximal. Se identificaron las estructuras anatómicas relevantes y se llevó a cabo la técnica quirúrgica "paso a paso". Se hizo énfasis en el hecho de presentar estructura ósea y partes blandas para el tornillo de calcar, sin complicaciones en el pasaje por el clavo. Se identificó para importancia de un paso explicado en el brochure, pero poco considerado en el "paso a paso" (avellanado de tornillo de calcar) asociado a una complicación menor (fractura superficie cortical con el pasaje del tornillo). Se realizó un primer procedimiento quirúrgico a través de condiciones similares a las simuladas, con excelentes resultados. Se identificó el éxito del avellanado, evitando la fractura de la superficie cortical, pero se identificó un nuevo paso crítico, el cual es el pasaje de las suturas por el tornillo de calcar. Al avellanar, el tornillo logra entrar en profundidad con su cabeza de bajo perfil, soltando las amarras del manguito rotador en el caso de profundizar el tornillo más allá de la cortical. El tornillo se retiró parcialmente y se restauraron las amarras sin complicaciones. El segundo paciente presentó un acto quirúrgico sin complicaciones, a través de un abordaje de menor dimensión y en un menor tiempo operatorio.

8. Evaluación crítica de los primeros casos de cirugía real y evaluar la necesidad de volver a pasos previos (Figuras 3 y 4): se realizó una evaluación crítica por el equipo quirúrgico y se registraron todas las ventajas/desventajas y detalles de ambos actos quirúrgicos para casos posteriores.

\section{DISCUSIÓN}

Se presenta un diseño de boot camp con tal de facilitar la transferencia desde el instructivo del nuevo implante brochure hasta la aplicación directa en un paciente. El campamento de entrenamiento presentó resultados excelentes, finalizando en dos actos quirúrgicos libres de complicaciones y seguros para nuestros pacientes.

El proceso de simulación planteado abarca desde una evaluación teórica del implante hasta la simulación en material artificial y cadavérico, previo a su utilización en casos reales. El hecho de discutir las características del implante y evaluarlas con relación a la evidencia disponible y la experiencia de los cirujanos es un acto dirigido a precisar la indicación y mejorar la seguridad del acto quirúrgico final en nuestros pacientes. Entender las nuevas características del implante permite planificar la simulación y el acto quirúrgico definitivo.

La simulación en material artificial y cadavérico disminuye las posibles complicaciones en el acto quirúrgico real. El hecho de realizar una técnica quirúrgica "paso a paso" desde la teoría, brochure y experiencia previa permite predecir posibles complicaciones o desafíos técnicos en la cirugía real. Sin embargo, el momento de la simulación es clave en demostrar desafíos no predecibles con el sólo hecho de una planificación teórica. La simulación en material artificial tiene por objetivo evaluar el uso del instrumental en una situación real y simular a baja fidelidad la utilización del nuevo implante, sin considerar el rol clave de las partes blandas en su colocación. Finalmente, en la simulación cadavérica, se identifican los detalles finales del acto quirúrgico, imposibles de planificar en los pasos previos. Es claramente la situación más cercana a la realidad y representa el acto culminante de la simulación en la aplicación de nuevos implantes en ortopedia y traumatología. 
La aplicación del nuevo implante en la cirugía definitiva no significa el término del proceso evaluativo. El análisis detallado del acto quirúrgico, la retroalimentación, la experiencia del equipo quirúrgico y la identificación de
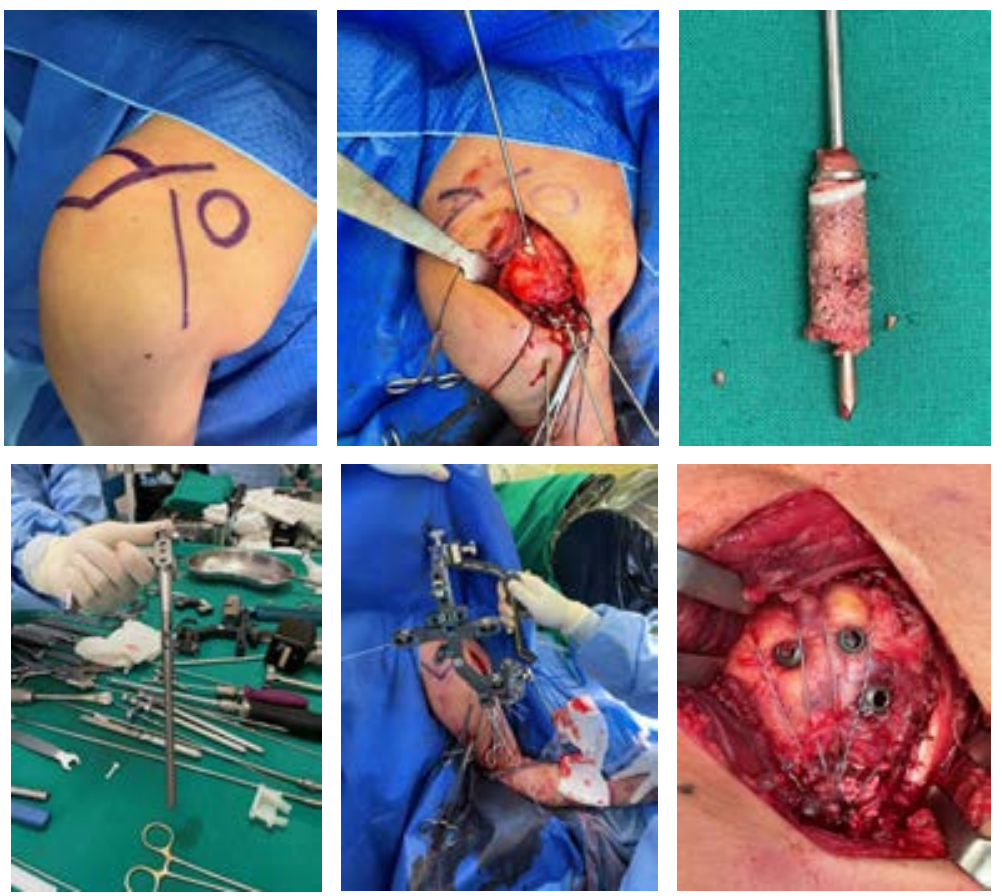

Figura 3: Resumen fotográfico de primera cirugía real.
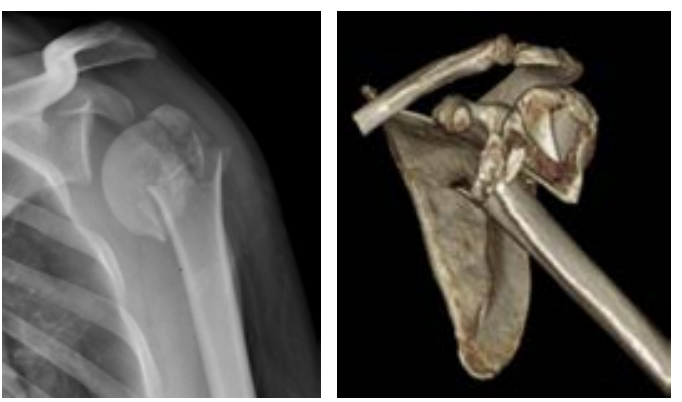

Figura 4:

Evaluación crítica del primer y segundo caso de cirugía real. situaciones no predecibles por las simulaciones previas es esencial para disminuir posibles complicaciones y realizar una curva de aprendizaje segura y rápida.

El uso de la simulación en la adquisición de habilidades quirúrgicas en ortopedia y traumatología ha demostrado ser eficaz. ${ }^{11}$ Una forma eficiente y rápida para obtener habilidades técnicas específicas de la cirugía ortopédica, sin poner en riesgo a los cirujanos ni a los pacientes son los boot camps. Se han utilizado campamentos de entrenamiento demostrando mejoras significativas y retención de habilidades cognitivas y de técnica quirúrgica específica en diversos contextos. ${ }^{13-15}$

El hecho de establecer un campamento de entrenamiento estructurado para la evaluación de nuevos implantes en ortopedia y traumatología es novedoso y no existen publicaciones previas al respecto. Creemos que esta pauta de trabajo puede ser mejorada y evaluada a través del método científico con tal de aumentar su eficiencia y utilidad con el fin de mejorar la adquisición de habilidades quirúrgicas con relación a nuevos implantes, aumentar la seguridad del paciente y disminuir la curva de aprendizaje y lo que ésta conlleva.

\section{REFERENCIAS}

1. Lee DH, Reasoner K, Stewart A. From concept to counter: a review of bringing an orthopaedic implant to market. J Am Acad Orthop Surg. 2015; 28 (14): e604-e611.

2. Roberts TT, Prummer CM, Papaliodis DN, Uhl RL, Wagner TA. History of the orthopedic screw. Orthopedics. 2013; 36 (1): 12-14.

3. Navarro M, Michiardi A, Castaño O, Planell JA. Biomaterials in orthopaedics. J R Soc Interface. 2008; 5 (27): 1137-1158.

4. Wilson NA, Schneller ES, Montgomery K, Bozic KJ. Hip and knee implants: current trends and policy considerations. Health Aff (Millwood). 2008; 27 (6): 1587-1598.

5. Sarpong NO, Herndon CL, Held MB, Neuwirth AL, Hickernell TR, Geller JA, et al. What Is the learning curve for new technologies in total joint arthroplasty? a review. Curr Rev Musculoskelet Med. 2020; 13 (6): 675-679.

6. Gofton WT, Solomon M, Gofton T, Pagé A, Kim PR, Netting C, et al. What do reported learning curves mean for orthopaedic surgeons? Instr Course Lect. 2016; 65: 633-643.

7. Wang Q, Goswami K, Shohat N, Aalirezaie A, Manrique J, Parvizi J. Longer operative time results in a higher rate of subsequent periprosthetic joint infection in patients undergoing primary joint arthroplasty. J Arthroplasty. 2019; 34 (5): 947-953. 
8. Sonnadara RR, Van Vliet A, Safir O, Alman B, Ferguson P, Kraemer W, et al. Orthopedic boot camp: examining the effectiveness of an intensive surgical skills course. Surgery. 2011; 149 (6): 745-749.

9. Chikwe J, de Souza AC, Pepper JR. No time to train the surgeons. BMJ. 2004; 328 (7437): 418-419.

10. Murphy JG, Torsher LC, Dunn WF. Simulation medicine in intensive care and coronary care education. J Crit Care. 2007; 22 (1): 51-55.

11. Akhtar KS, Chen A, Standfield NJ, Gupte CM. The role of simulation in developing surgical skills. Curr Rev Musculoskelet Med. 2014; 7 (2): 155-160.

12. Katthagen JC, Schwarze M, Bauer L, Meyer-Kobbe J, Voigt $C$, Hurschler $C$, et al. Is there any advantage in placing an additional calcar screw in locked nailing of proximal humeral fractures? Orthop Traumatol Surg Res. 2015; 101 (4): 431-435.

13. Fernandez GL, Page DW, Coe NP, Lee PC, Patterson LA, Skylizard L, et al. Boot cAMP: educational outcomes after 4 successive years of preparatory simulationbased training at onset of internship. J Surg Educ. 2012; 69 (2): 242-248.

14. Moazed F, Cohen ER, Furiasse N, Singer B, Corbridge TC, McGaghie WC, et al. Retention of critical care skills after simulation-based mastery learning. J Grad Med Educ. 2013; 5 (3): 458-463.

15. Cohen ER, Barsuk JH, Moazed F, Caprio T, Didwania A, McGaghie WC, et al. Making July safer: simulationbased mastery learning during intern boot camp. Acad Med. 2013; 88 (2): 233-239.

Financiamiento: Ningún autor, su familia inmediata y cualquier fundación de investigación a la que estén afiliados recibieron pagos económicos u otros beneficios de ninguna entidad comercial relacionada con el tema de este artículo. Rodrigo Liendo y Francisco Soza han impartido charlas educativas financiadas por Zimmer-Biomet relacionadas con el tema de este artículo.

\author{
Correspondencia: \\ Julio J Contreras \\ Diagonal Paraguay \#362, PC 8330077, \\ Santiago, Chile (+569 79981070). \\ E-mail: juliocontrerasmd@gmail.com
}

